\title{
Simulative Investigation of Coherent Optical OFDM Communication with Gbits/s Data Rates
}

\author{
R.S. Asha and Dr. Jayasree V.K.
}

\begin{abstract}
CO-OFDM has the advantages of both 'coherent detection' and 'OFDM modulation' and provides many merits for future high-speed fiber transmission systems. In this paper, we analyze the performance of Coherent Optical OFDM (CO-OFDM) for different data rates like 1Gbps, 20Gbps and 100Gbps. 4 QAM-OFDM is generated, modulated using MZM and transmitted through the fiber for a distance of $250 \mathrm{~km}$. Due to the different nonlinearities of fiber the signal is distorted. At the receiver end the filtered optical signal is converted to electrical signal and OFDM is demodulated. Results shows that at the receiver as the data rate increases, the distortions in the OFDM spectrum and QAM constellations also increase.
\end{abstract}

Keywords--- OFDM- Orthogonal Frequency Division Multiplexing, Gbps-Giga Bits per Second, MZM- MachZehnder Modulator, QAM- Quadrature Amplitude Modulation

\section{INTRODUCTION}

$\mathrm{O}$ RTHOGONAL frequency division multiplexing (OFDM) is used in different digital standards for broad-range of applications like digital broadcasting and wire-line and wireless communication systems [1]. OFDM can be applied in optical long haul transmission systems and has many advantages over single-carrier modulation format [2]-[4]. Many advantages of the OFDM technique have been demonstrated in the communication sector. Mainly, the frequency spectra of OFDM subcarriers are partially overlapped, resulting in high spectral efficiency. Secondly, the channel dispersion of the transmission system can be easily estimated and removed, and thirdly the signal processing in the OFDM transceiver can utilize the advantage of the efficiency of the FFT/IFFT algorithm which has low computation complexity. Coherent optical OFDM (COOFDM) has been proposed as an equivalent optical-domain multi-carrier format for long haul transmission [2]. Incoherent Optical OFDM (IO-OFDM) has also been proposed with similar dispersion tolerance and much simpler detection scheme [3].

But, the CO-OFDM is superior to IO-OFDM in spectral efficiency, OSNR requirement, and Polarization Mode Dispersion insensitivity. OFDM is generally susceptible to nonlinearity and phase noise because of its high peak to average power ratio (PAPR) [1]. Therefore it is very important

R.S. Asha, Research Scholar, Department of Electronics, Model Engineering College, Thrikkakara. E-mail:asharspillai@gmail.com

Dr. Jayasree V.K., HOD, Department of Electronics, Model Engineering College, Ernakulam

DOI: 10.9756/BIJRCE.10426 to investigate and improve the CO-OFDM system transmission performance including fiber nonlinearity and receiver nonlinearity, in order to ascertain its suitability for optical transmission.

Optical OFDM (O-OFDM) systems provide an accurate solution for $100 \mathrm{~Gb} / \mathrm{s}$ Ethernet data traffic [5]-[8]. Latest results show the transmission of Tbit/s data rates over a fiber length of $600 \mathrm{~km}$ [9]. But the long-haul optical systems are affected by fiber nonlinearity if the optical powers are kept low [10]. This affects the design of long-haul systems; low launch powers reduces the optical signal-to-noise ratio (OSNR) of the given link, thereby limiting the size of the constellation that can be used [11]. Therefore, it is essential to reduce fiber nonlinearity for achieving higher spectral efficiencies in long-haul transmission [11], [12].

CO-OFDM uses different subcarriers in parallel data streams. The M-ary Quadrature Amplitude Modulation (QAM) or Phase Shift Keying (PSK) is used to modulate the subcarriers before modulating with a high frequency microwave carrier. The multicarrier modulation provides strong vigorousness to intersymbol interferences because the duration of symbol is extremely longer than the root-meansquare (RMS) delay of the optical wireless channel. Therefore, O-OFDM multilevel quadrature amplitude modulation (M-QAM) encourages very high data rates [13][15]. In the optical communication system, the data is optically modulated and it should be unipolar (15).

High spectral efficiency modulation formats are used for the increasing demand of the channel capacity in optical communications [16]. O-OFDM has been proved to be suitable for 100 plus Gbps systems and CO-OFDM is considered as a emerging technology in future long-haul optical communication networks because of high spectral efficiencies, resistance to polarization dispersion, adjustable bandwidth scalability and allocation [17]. But, an important drawback of the OFDM system is its susceptibility to fiber nonlinearity due to its high peak-to- average power ratio (PAPR) [18].

\section{THEORETICAL FUNDAMENTALS FOR CO-OFDM}

\section{A. Principle of Orthogonal Frequency-Division Multiplexing (OFDM)}

OFDM is a special form of multi-carrier modulation (MCM), an implementation of which is depicted in Fig. 1. The structure of a complex IQ modulator/demodulator, which is normally used in MCM systems, is also shown in the figure. The MCM transmitted signal ' $S(t)$ ' is represented as 


$$
\begin{gathered}
\mathrm{S}(\mathrm{t})=\sum_{\mathrm{i}=-\infty}^{+\infty} \sum_{\mathrm{k}=1}^{\mathrm{N}_{\mathrm{sc}}} \mathrm{C}_{\mathrm{ki}} \mathrm{S}_{\mathrm{k}}\left(\mathrm{t}-\mathrm{iT}_{\mathrm{s}}\right) \\
S_{k}(t)=\prod(t) \exp \left(6 \pi f_{k}(t)\right. \\
\Pi(t)=\left\{\begin{array}{c}
1,\left(0<t \leq \mathrm{T}_{\mathrm{s}}\right. \\
0,\left(\mathrm{t} \leq 0, \mathrm{t}>\mathrm{T}_{\mathrm{s}}\right.
\end{array}\right\}
\end{gathered}
$$

Where ' $\mathrm{C}_{\mathrm{ki}}$ ' is the $\mathrm{i}^{\text {th }}$ information symbol at the $\mathrm{k}^{\text {th }}$ subcarrier, $S_{k}$ is the waveform for the $\mathrm{k}^{\text {th }}$ subcarrier, ' $\mathrm{N}_{\mathrm{sc}}$ ' is the number of subcarriers, ' $f_{k}$ ' is the frequency of the subcarrier, and ' $T$ ' is the symbol period. The optimum detector for each subcarrier can use a filter that matches the subcarrier waveform, or a correlator matched to the subcarrier as in Fig. 1. Therefore, the detected information symbol ' $\mathrm{C}_{\mathrm{ik}}$ "' at the output of the correlator is given by

$$
\begin{gathered}
C_{k i}^{\prime}=\int_{0}^{T_{s}} r\left(t-i T_{s}\right) S_{k}^{*} d(t) \\
=\int_{0}^{T_{s}} r\left(t-i T_{s}\right) \exp \left(-j 2 \pi f_{k} t\right) d t
\end{gathered}
$$

Where ' $r(t)$ ' is the received time-domain signal. The basic MCM uses non-overlapped band limited signals, and can implemented using a bank of large number of oscillators and filters at both transmit and receive end. The major disadvantage of MCM is that it requires large bandwidth. In order to design the filters and oscillators cost-efficiently, the channel spacing should be multiples of the symbol rate which in turn reduces the spectral efficiency.

A novel approach called OFDM is investigated including overlapped but orthogonal signal set [19]-[20]. This orthogonality generates from the straightforward correlation between any two subcarriers, given by

$$
\begin{gathered}
\delta_{u}^{\mathrm{t}}=\frac{1}{T_{s}} \int_{0}^{T_{s}} S_{k} S_{l}^{*} d t \\
=\frac{1}{T_{s}} \int_{0}^{T_{s}} \operatorname{exp!}\left(j 2 \pi\left(f_{k}-f_{l}\right) t\right) d t \\
=\exp \left(\pi / f_{k}-f_{l}\right) T_{s} \sin \frac{\left(\pi\left(f_{k}-f_{l}\right) T_{s}\right.}{\pi\left(f_{k}-f_{l}\right) T_{s}}
\end{gathered}
$$

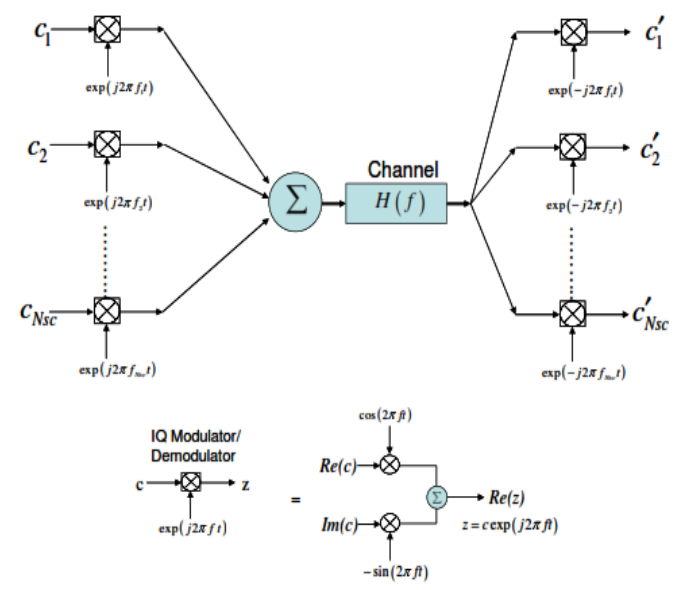

Fig. 1: Generic Implementation of Multi Carrier Modulation (MCM) System
It can be seen that if the following condition

$$
\mathrm{f}_{\mathrm{k}}-\mathrm{f}_{\mathrm{l}}=\mathrm{m} \frac{1}{\mathrm{~T}_{\mathrm{s}}}
$$

is satisfied, then the two subcarriers are orthogonal to each other. This implies that these orthogonal subcarrier sets, with their frequencies spaced at multiple of inverse of the symbol rate can be recovered with the matched filters (Eq. 4) without inter-carrier interference (ICI), in spite of strong signal spectral overlapping.

\section{B. Principle of Coherent Optical OFDM}

Because of its strong resistance to the channel dispersion, OFDM is actively utilized for applications in the optical domain. The most critical assumption for OFDM is the linearity in modulation, transmission, and demodulation. As a result, a linear transformation is the key goal for the OFDM implementation. A generic optical OFDM system can be divided into five functional blocks including (i) the RF OFDM transmitter, (ii) the RF-to-optical (RTO) up-converter, (iii) the optical channel, (iv) the optical-to-RF (OTR) down-converter, and (v) the RF OFDM receiver [21]. The RF OFDM transmitter/receiver generates/recovers the OFDM signals either in baseband or RF band. It is apparent that the challenges for CO-OFDM implementation are to obtain a linear RTO up-converter and linear OTR down-converter.

It has been analyzed that by biasing the Mach-Zehnder modulators (MZMs) at null point, a linear conversion between the transformation from optical field signal to RF (or electrical) signal can be achieved [21], [23]-[24]. Now by putting together such a composite system cross RF and optical domain [21]-[22], a linear channel can be constructed where OFDM can perform its best, i.e., mitigating channel dispersion impairment, in both RF domain and optical domain.

\section{Optical Spectral Efficiency for CO-OFDM}

In CO-OFDM systems, ' $N_{s c}$ ' subcarriers are transmitted in every OFDM symbol period of ' $T T_{s}$ ', the total symbol rate ' $R$ ' for CO-OFDM systems is given by

$$
\mathrm{R}=\frac{\mathrm{N}_{\mathrm{sc}}}{\mathrm{T}_{\mathrm{s}}}
$$

The OFDM bandwidth ' $B_{\text {OFDM }}$ ' is given by

$$
B_{O F D M}=\frac{2}{T_{s}}+\frac{N_{S c}-1}{t_{s}}
$$

Where ' $t_{s}$ ' is the observation period. If a large number of subcarriers are used, the bandwidth efficiency of OFDM ' $\eta$ ' is found to be

$$
\eta=2 \frac{R}{B_{O F D M}}=2 \alpha, \alpha=\frac{t_{s}}{T_{s}}
$$

The factor of ' 2 ' represents for two polarizations in the fiber. Using a typical value of 8/9 [19], we obtain the optical Spectral efficiency factor $\eta$ of $1.8 \mathrm{Baud} / \mathrm{Hz}$. The optical spectral efficiency gives $3.6 \mathrm{bit} / \mathrm{Hz}$ if QPSK modulation is used for each subcarrier. The spectral efficiency can be further improved by using higher-order QAM modulation [25]. 
Table 1: Simulation Parameters

\begin{tabular}{|l|l|}
\hline Laser frequency & $193.1 \mathrm{THz}$ \\
\hline Laser power & $10 \mathrm{dBm}$ \\
\hline Fiber Attenuation & $0.2 \mathrm{~dB} / \mathrm{km}$ \\
\hline Fiber length & $250 \mathrm{~km}$ \\
\hline Fiber dispersion & $16.75 \mathrm{ps} / \mathrm{nm} / \mathrm{km}$ \\
\hline $\begin{array}{l}\text { Differential group } \\
\text { delay }\end{array}$ & $0.2 \mathrm{ps} / \mathrm{km}$ \\
\hline $\begin{array}{l}\text { Max. nonlinear } \\
\text { phase shift }\end{array}$ & $3 \mathrm{mrad}$ \\
\hline
\end{tabular}

In the RF OFDM transmitter, the input data is split into multiple parallel branches. This is called as "serial-to-parallel" conversion. The number of the multiple branches equals to the number of subcarriers. Then the converted signal is mapped on to quadrature amplitude modulation (QAM). The IFFT is used to convert the mapped signal from frequency domain into time domain. Two-dimensional complex signal is used to carry the information. A pair of electrical low-pass filters is used to remove the alias sideband signal.

At the RTO up-converter, the baseband OFDM signal is up shifted onto optical domain using an optical I/Q modulator, which is comprised by two Mach-Zehnder modulators (MZMs) with a $90^{\circ}$ optical phase shifter. The unconverted OFDM signal in optical domain is given by

$$
E(t)=\exp \left(j \omega_{L D 1} t+{ }_{L D 1}\right) S_{B}(t)
$$

Where ' $\omega_{L D 1}$ 'and ' $\phi_{L D 1}$ ' are the frequency and phase of the transmitter laser, respectively and $S_{B}{ }^{(t)}$ is the baseband OFDM. The optical signal ' $E$ (t)' is applied into the optical fiber link, with an impulse response of ' $h(t)$ '. The received optical signal 'E' ( $\mathrm{t}$ )' becomes

$$
E^{\prime}(t)=\exp \left(j \omega_{L D 1} t+{ }_{L D 1}\right) S_{B}(t) * \mathrm{~h}(\mathrm{t})
$$

Table 2: Simulation Parameters

\begin{tabular}{|l|l|}
\hline Photodetector responsivity & $1 \mathrm{~A} / \mathrm{w}$ \\
\hline Photodetector dark current & $10 \mathrm{nA}$ \\
\hline Photodetector center frequency & $193.1 \mathrm{THz}$ \\
\hline Fiber length & $250 \mathrm{~km}$ \\
\hline Photodetector sample rate & $5 \mathrm{~Hz}$ \\
\hline Photodetector thermal noise & $10^{-22} \mathrm{w} / \mathrm{Hz}$ \\
\hline
\end{tabular}

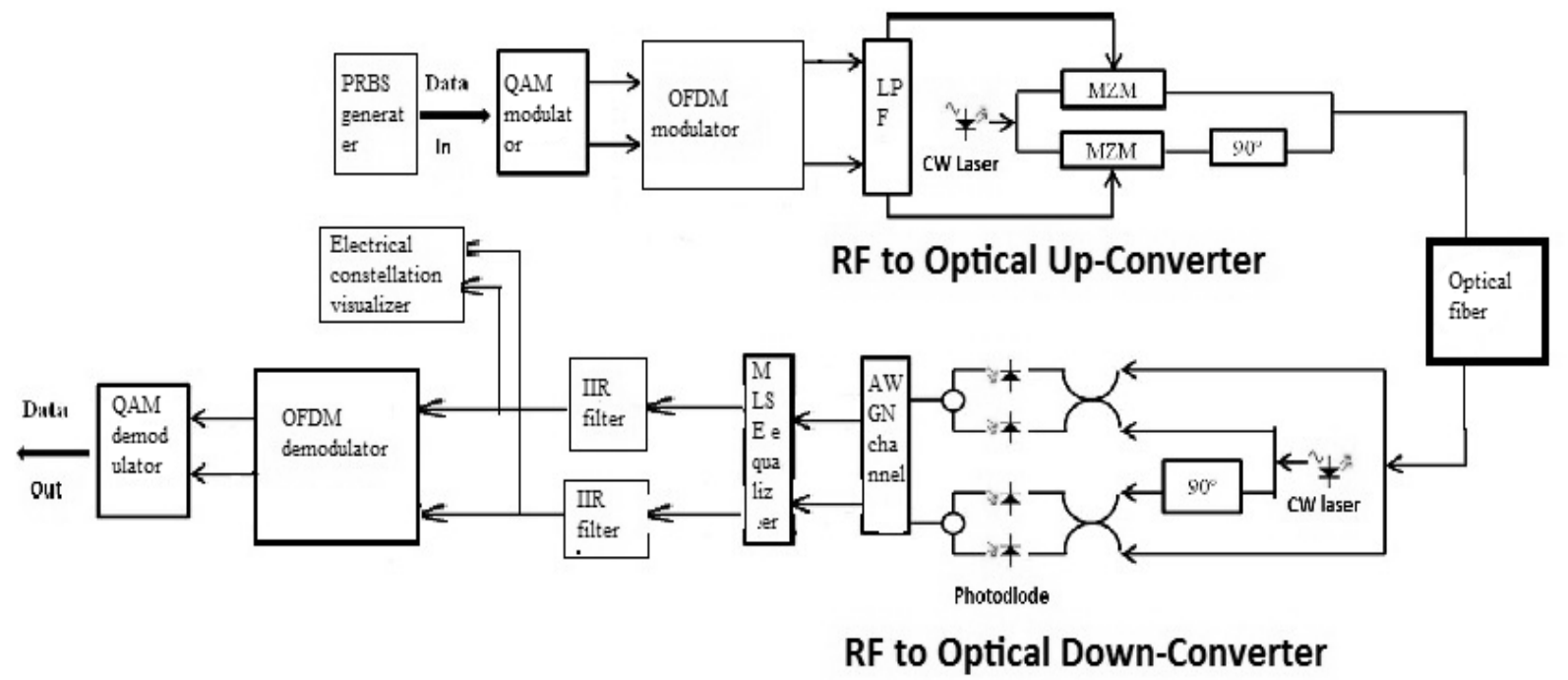

Fig. 2: Block Diagram of Coherent Transmitter and Receiver

룹

OSA

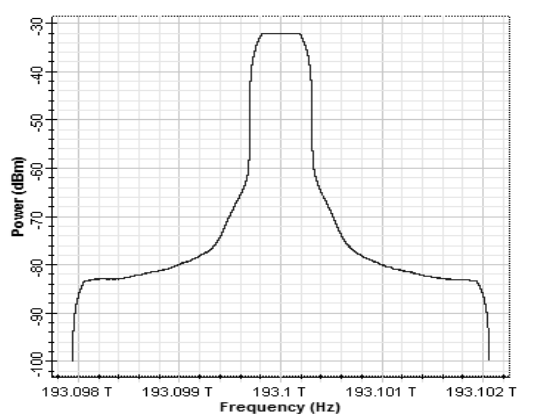

Fig. 3: 1 Gbps OFDM MZM Generated Output

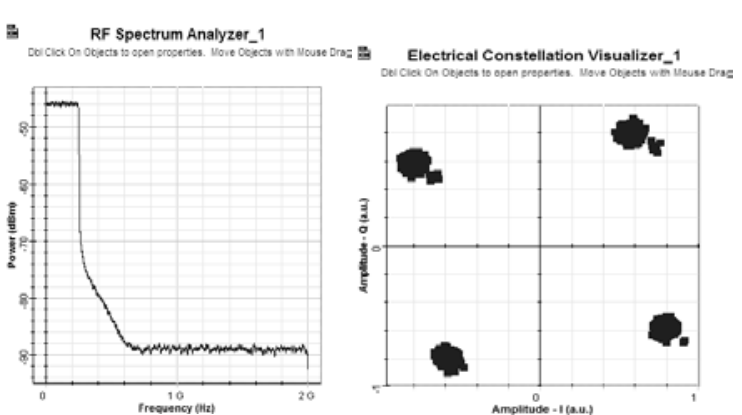

Fig. 4: (a) 1Gbps OFDM Demodulator in-phase Output.

(b) 1Gbps 4-QAM CO-OFDM Received Constellation Diagram 


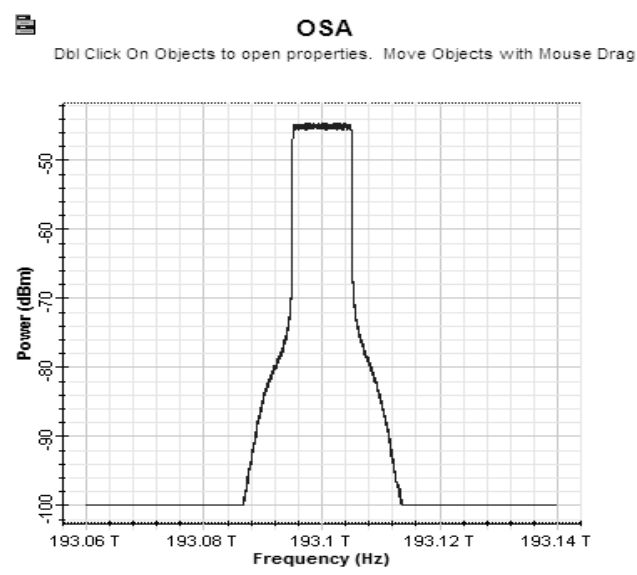

Fig. 5: 20Gbps OFDM Modulated MZM Output

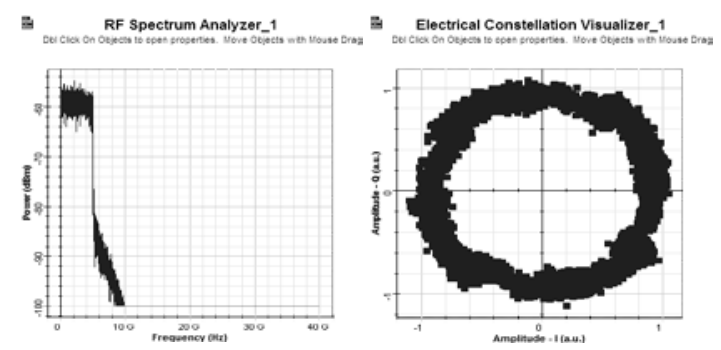

Fig. 6: (a) 20 Gbps OFDM Demodulator in-phase Output.

(b) 20 Gbps CO-OFDM Received Constellation Diagram

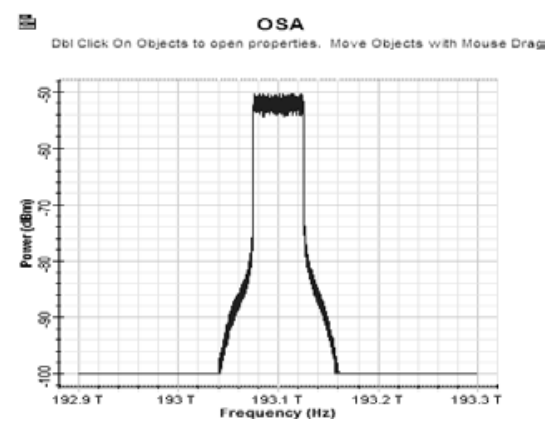

Fig. 7: 100Gbps 4-QAM OFDM Generated Output

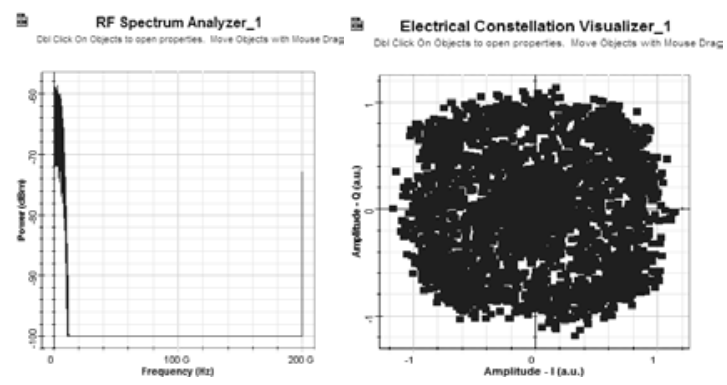

Fig.8: (a) 100Gbps Demodulated OFDM Output. (b) 100Gbps 4 QAM -OFDM Received Constellation Diagram

\section{RESULTS AND DISCUSSION}

Figure 3 and 4 show the received plot of the constellation spread of amplitudes and phases in a conventional 4 QAM CO-OFDM system for datarate of $1 \mathrm{Gbps}$. It shows that the constellations of the QAM symbols are tightly grouped. Fig.5and 6 shows the received plot of the constellation spread of amplitude and phases in a 4 QAM CO-OFDM with 20Gbps datarate. Fig.7and 8 shows the generated 100Gbps 4 QAMCO-OFDM spectrum and received constellation spread of 100Gbps datarate. The phase noise is caused by fiber nonlinearity induced inter-carrier interference (ICI).

Results show that as data rates increases, the amplitude and phase noises of the received OFDM spectrum system are also increases. This is due to the nonlinearities in the optical fiber, shot noise and thermal noise of photo detectors at the receiver.

\section{CONCLUSION}

The performance of Coherent Optical OFDM (CO-OFDM) is analyzed for different data rates such as $1 \mathrm{Gbps}$, 20Gbps and 100Gbps. 4 QAM-OFDM is modulated using MZM and transmitted through the fiber for a distance of $250 \mathrm{~km}$. Due to the different nonlinearities of fiber and photo detector, the signal is distorted. At the receiver end the filtered optical signal is converted to electrical signal and OFDM is demodulated. Results show that at the receiver, as the data rates increases the distortions of the OFDM spectrum and QAM constellations also increases.

\section{REFERENCE}

[1] S.L.Jansen, I.Morita, T.C.M.Schenk and H.Tanaka, "121.9-Gb/PDMOFDM transmission with $2-\mathrm{b} / \mathrm{s} / \mathrm{Hz}$ spectral efficiency over $1000 \mathrm{~km}$ of SSMF,” J. Lightw. Technol., vol. 27, no. 3, pp. 177-188, Feb.2009.

[2] Takahashi, A.AlAmin, S.L, Jansen, I.Morita, and H.Tanaka, "DWDM transmission with 7.0-bit/s/Hz spectral efficiency using 865.1-Gbit/s coherent PDM-OFDM signals," in Proc. Opt. Fiber Commun. Conf., San Diego, pp. 1-3, 2009.

[3] B. J. C. Schmidt, Z. Zan, L. B. Du, and A. J. Lowery, "120 Gbit/sover 500-km using single-band polarization-multiplexed self-coherent optical OFDM,” J. Lightw. Technol., vol. 28, no. 4, pp. 328-335, Feb. 2010.

[4] Yang,Y.Tang,Y.Ma,andW.Shieh,"Experimentaldemonstration and numerical simulation of $107-\mathrm{Gb} / \mathrm{s}$ high spectral efficiency coherent optical OFDM,” J. Lightw. Technol., vol. 27, no. 3, pp. 168-176, Feb.2009.

[5] Y. Ma, Q. Yang, Y. Tang, S. Chen, and W. Shieh, "1-Tb/s singlechannel coherent optical OFDM transmission with orthogonal-band multiplexing and subwavelength bandwidth access," J. Lightw. Technol., vol. 28, no. 4, pp. 308-315, Feb. 2010.

[6] A.J.Lowery, L.B.Du and J.Armstrong, "Performance of optical OFDM in ultralong-haul WDM lightwave systems," J. Lightw.Technol., vol. 25, no. 1, pp. 131-138, Jan. 2007.

[7] R.J.Essiambre, G.J.Foschini, G.Kramer and P.J.Winzer, “Capacity limits of information transport in fiber-optic networks,” Phys. Rev. Lett., vol. 101, pp. 163901-1-163901-4, 2008.

[8] A. D. Ellis, J. Zhao, and D. Cotter, "Approaching the non-linear Shannon limit,” J. Lightw. Technol., vol. 28, no. 4, pp. 423-433, Feb. 2010.

[9] B S. Hara and R. Prasad, Multicarrier Techniques for 4G Mobile Commmications, (Artech House, Boston, 2003).

[10] W. Shieh and C. Athaudage, "Coherent optical orthogonal frequency division multiplexing,” Electron. Lett.,vol 42, pp.587 - 589,May 2006.

[11] J. Lowery, L. Du, and J. Armstrong, "Orthogonal frequency division multiplexing for adaptive dispersion compensation in long haul WDM systems," in Optical Fiber Communication Conference and Exposition 
and The National Fiber Optic Engineers Conference, Technical Digest, (Anaheim, CA, USA), Paper PDP39,2006.

[12] I. B. Djordjevic and B. Vasic, "Orthogonal frequency division multiplexing for high-speed optical transmission,” Opt. Express, vol.4, Pp.3767-3775, 2006.

[13] C S. Dimitrov and H. Haas, "Information Rate of OFDM Based Optical Wireless Communication Systems with Nonlinear Distortion," IEEE Journal of Lightwave Technology, vol. 31, no. 6, 2, Pp. 918-929, 2013.

[14] H. Elgala, R. Mesleh, H. Haas and B. Pricope, "OFDM Visible Light Wireless Communication Based on White LEDs," Vehicular Technology Conference, Dublin, Pp. 2185-2189, April-2007.

[15] S. Dimitrov, S. Sinanovic and H. Haas, "Signal Shaping and Modulation for Optical Wireless Communication,"IEEE Journal of Lightwave Technology, vol. 30, no. 9, Pp. 1319-1328, 2012.

[16] P. J. Winzer, "Beyond 100G ethernet," IEEE Commun. Mag. vol.48,no.7, pp. 26-30, 2010.

[17] S. L. Jansen, I. Morita, T. C. W. Schenk and H.Tanaka,“121.9-Gb/s PDM-OFDM Transmission with 2-b/s/Hz Spectral Efficiency Over 1000km of SSMF,” J. Light w. Technol., vol. 27, pp. 177-188, 2009.

[18] J. Armstrong, “OFDM for optical communications," Journal of Light wave technology 27, Pp. 189-204, 2009.

[19] R. W. Chang, "Synthesis of band-limited orthogonal signals for multichannel data transmission,” Bell Sys.Tech. J.vol. 45, pp.1775-1796, 1966.

[20] B. R. Saltzberg, "Performance of an efficient parallel data transmission system,” IEEE Trans. Commun,.vol.15, pp.805-813, 1967.

[21] W. Shieh and C. Athaudage, "Coherent optical orthogonal frequency division multiplexing,” Electron. Lett.,vol. 42, pp.587-589, 2006.

[22] Y. Tang, W. Shieh, X. Yi and R. Evans, "Optimum design for RF-tooptical up-converter in coherent optical OFDM systems,” IEEE Photon. Technol. Lett.,vol. 19, pp. 483 - 485, 2007.

[23] D. S. Ly-Gagnon, S. Tsukarnoto, K. Katoh, and K. Kikuchi, "Coherent detection of optical quadrature phase-shift keying signals with carrier phase estimation,” J. Light wave Technol.,vol. 24, pp.12-21, 2006.

[24] S. J. Savory, G. Gavioli, R. I. Killey, and P. Bayvel, "Electronic compensation of chromatic dispersion using a digital coherent receiver," Opt. Express,vol. 15, pp.2120-2126, 2007.

[25] Arthur James Lowery and Jean Armstrong, "Orthogonal-frequencydivision multiplexing for dispersion compensation of long-haul optical systems”, Optic express, vol.14, no.6, pp. 2079-2084, March 2006.

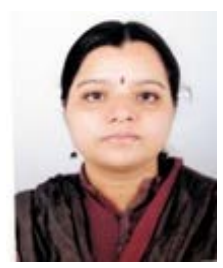

Asha.R.S. received M.E degree in Communication Systems from Anna University Chennai,India in 2008. Currently she is doing $\mathrm{PhD}$ in Model Engineering College (Research Center,CUSAT),Kerala,India. Her research areas are Radio over Communication, Optical Signal processing, Free space communication and Wireless Communication

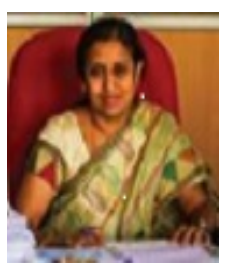

Jayasree V.K. received M.Tech degree in Biomedical Engineering from Indian Institute Technology, Chennai, India in 1998 and $\mathrm{PhD}$ degree from Cochin University of Science and Technology, India in 2009. Currently she is Head of the Department of Electronics, Govt. Model Engineering College, Cochin University of Science and Technology, India. Her research interests include biomedical image processing and optical communication techniques 